\title{
Un enfoque óptimo para la distribución de energía de una micro-red usando Control Predictivo basado en Modelo (MPC): una simulación de un caso de estudio
}

César Hernández, Francisco Rodríguez, José Carlos Moreno

Departamento de Informática, Campus de Excelencia Internacional Agroalimentario ceiA3, Centro de Investigación en Energía Solar CIESOL, Universidad de Almería, 04120, España, e-mail: \{chdezh,frrodrig,jcmoreno\}@ual.es Paulo Renato da Costa Mendes, Julio Elias Normey-Rico

Universidad de Santa Catarina, Florianopolis, Brasil, e-mail: paulorcm@hotmail.com, julio.normey@ufsc.br

\section{Resumen}

Las micro-redes permiten la integración de fuentes de energía renovables como fuentes de energía solar y eólica, también permiten la integración de sistemas distribuidos tales como producción combinada de calor y energía y almacenamiento de energía. Además, el uso de las fuentes locales de energía para servir cargas locales ayuda a reducir las pérdidas de energía en la transmisión y distribución, aumentando aún más la eficiencia del sistema de suministro eléctrico. En este trabajo, el problema de optimización de la energía dentro de una micro-red de energía renovable (MG) con un sistema de almacenamiento de energía (ESS), que intercambia energía con la red principal se desarrolla y se resuelve con el uso de técnicas de control predictivo basado en modelo (MPC). El modelado del sistema utiliza la metodología de los Energy Hubs. Las técnicas de MPC permiten maximizar el beneficio económico de la micro-red y reducir al minimo la degradación del sistema de almacenamiento.

Palabras clave: Energías renovables; Energy Hubs; Micro-red; Control Predictivo basado en Modelo.

\section{Introducción}

Hoy en día y en el futuro, los sistemas de generación y distribución de energía eléctrica deberán ser distribuidos, inteligentes, renovables y adaptables; estos requerimientos conducirán a la evolución de una red eléctrica convencional hacia futuras redes inteligentes.

Una micro-red puede proveer una distribución optima de energía para sus consumidores mediante el uso de estrategias de control. Hoy en día, el concepto de MG se centra principalmente en la integración de fuentes distribuidas de energía renovable, almacenamiento en baterías y metodologías para la administración y control de la energía, como se muestra en [15], [3], [16], [20], [5], [14].

En las micro-redes se integran sistemas de energía que se componen por fuentes de energía dis- tribuida y múltiples cargas eléctricas que operan como una red autónoma y que pueden estar a su vez conectadas a la red eléctrica en modo paralelo o en modo isla. Una MG puede ser considerada como una versión a pequeña escala de una red tradicional de energía [4]. Todas estas ventajas han motivado por un lado, a un incremento en el desarrollo de micro-redes en diversas áreas, tales como entornos tipo campus, operaciones militares y sistemas de comunidades. Por otro lado, han motivado al estudio de técnicas de optimización de energía en micro-redes.

En la literatura existe una gran cantidad de métodos y aplicaciones de MG, en [12] se presenta una estructura básica de una MG y se discuten técnicas de control. En [7], los autores realizan una revisión de los documentos más recientes que hablan sobre el uso de sistemas híbridos de almacenamiento de energía (HESS), los cuales facilitan la introducción de fuentes de energía renovables en micro-redes, así también, realizan un análisis y comparación del uso de HESS formados por diferentes sistemas de almacenamientos y diferentes topologías. En [21] se presenta un resumen de los enfoques (configuración del sistema, tamaño de la unidad, control y gestión de la energía) disponible y que actualmente se investigan para el diseño óptimo de sistemas híbridos RES. En [6] una estrategia de control para la integración de sistemas distribuidos de almacenamiento en una red fotovoltaica y que incluye cargas variables es desarrollada. El control propuesto permite usar la máxima energía fotovoltaica en diferentes condiciones de operación de la MG y permite una transferencia suave entre la conexión de red y el aislamiento. En general, la gestión de micro-redes se lleva a cabo por medio de algoritmos heurísticos [11], aunque hay aplicaciones que hacen uso de estrategias de MPC, como la que se presenta en [8] y [24]. El uso de estrategias de MPC ([2]) presenta buenas soluciones.

El objetivo de este artículo es presentar una simulación de un caso estudio de la distribución óptima de energía en una MG utilizando estrategias de control predictivo. El algoritmo que se propone permite optimizar el manejo de las fuentes de en- 
ergía renovable, la carga y descarga del sistema de almacenamiento y, la compra y venta de energía eléctrica de la red principal.

Este artículo está organizado de la siguiente manera: en la sección 2 se presentan la micro-red y la metodología utilizada para la distribución óptima de energía; en la sección 3 se presentan los resultados y discusiones y finalmente, en la sección 4 se presentan las conclusiones.

\section{Materiales y métodos}

La MG que se estudia en este artículo está integrada por un sistema fotovoltaico (PV), un vehículo eléctrico del cual sus baterías se usan como un sistema de almacenamiento, y dos sistemas de carga (un edificio y un invernadero). En la Figura 1 se muestra esta micro-red. Además, se considera que la micro-red se encuentra conectada con la red principal y que se permite la compra y venta de energía cuando sea necesario.

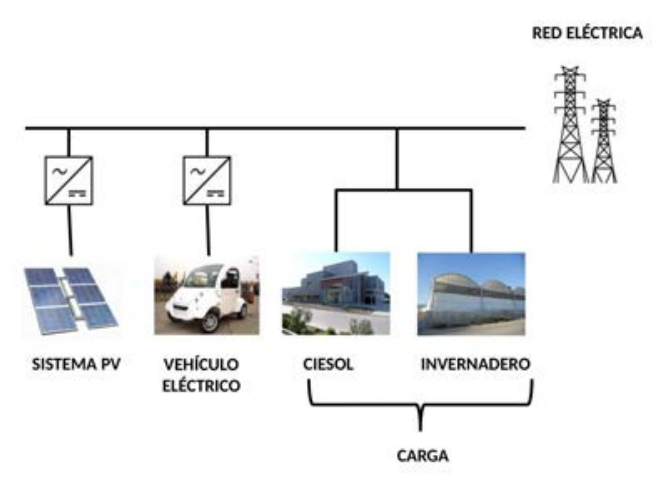

Figura 1: Micro-red.

\subsection{Materiales}

El invernadero estudiado en este artículo tiene una estructura tipo "Parral", una área de superficie de $877 \mathrm{~m}^{2}$, orientado en dirección N-S. Las instalaciones se encuentran ubicadas en la estación experimental Las Palmerillas de la Fundación Cajamar, en el municipio de El Ejido en el sureste de España [22]. El invernadero cuenta con sistema de ventilación lateral y cenital accionada por motores AC independientes, aerotermo, sistema de calefacción por tuberías de agua caliente alimentadas con biomasa, un sistema de enriquecimiento de $\mathrm{CO}_{2}$ creado por la quema de biomasa, mallas de sombreo y sistemas de alimentación de agua y nutrientes. El invernadero cuenta también, con un equipo de medida de 52 variables y está diseñado para el desarrollo de pruebas de identificación e implementación de estrategias de control climático. Además, cuenta con un sistema sensores de demanda de energía (SINEAX M 561) [23].

El edificio CDdI-CIESOL-ARFRISOL es un centro de investigación en energía solar localizado dentro del Campus de la Universidad de Almería en el sureste de España. Está distribuido en dos pisos con una superficie de $1071.92 \mathrm{~m}^{2}$, se compone por seis oficinas, todas las oficinas tienen orientación este y están situadas en la planta baja, con la excepción de la oficina principal, que se encuentra en el piso superior, ocho laboratorios que se orientan al norte (cuatro situados en la planta baja y dedicados a "Química Solar", y los otros cuatro se colocan en el piso superior, dos para el modelado y la unidad de control automático, y el restante para la evaluación de la unidad de recursos solares), una planta donde se encuentra una maquina de alta eficiencia de calefacción y absorción, y por último, cuenta también con una cocina y el cuarto de servicios.

El sistema PV se encuentra localizado en la terraza del edificio CIESOL. Es un sistema de $9.324 k W p$, con un número total de 4 módulos asociados en conjuntos de 14 paneles con una capacidad de $222 \mathrm{Wp} /$ panel, este sistema se encuentra conectado a 3 inversores de $2500 \mathrm{~W}$.

Las baterías con las que cuenta el vehículo eléctrico son del tipo de modelo Green Saver, con una capacidad total de $C=11 k W h$.

\subsection{Métodos}

La metodología que se usa en este artículo ha sido presentada en [10] y [9].

Un Energy $H u b$ se define como la interfaz entre la producción de energía, los consumidores y la línea de transmisión. Desde el punto de vista de un sistema de energía, un Energy Hub puede ser identificado como una unidad que proporciona las siguientes características: (1) una entrada y una salida de energía; (2) la conversión de energía; (3) un sistema de almacenamiento de energía. Algunas de las principales características de los Energy $H u b$ son

- Dentro de los Energy Hub, las pérdidas se producen sólo en elementos convertidores y almacenamiento.

- Si no se menciona explícitamente, se supone el flujo de energía unidireccional desde las entradas hasta las salidas de los convertidores.

- El flujo de energía a través de los Energy Hub se caracteriza solamente por la eficiencia en- 
ergética y la energía, no se utilizan otras cantidades.

Para describir la metodología se tiene que, por un lado una unidad de conversión convierte en cada instante de tiempo $k$, un flujo de entrada genérico $u_{r}^{L}(k)$ de un Hub en una salida genérica $y_{p}(k)$. Donde el superíndice $L$ se asocia al flujo de entrada del convertidor. La conversión de entrada-salida se define a través de factores de acoplamiento $\gamma_{p, r}^{L}$ que corresponden a la eficiencia de conversión del convertidor entre los flujos de entrada y salida:

$$
y_{p}(k)=\gamma_{p, r}^{L} u_{r}^{L}(k)
$$

Si se tienen múltiples convertidores, entonces se puede representar de la siguiente manera:

$$
\begin{aligned}
\underbrace{\left[\begin{array}{c}
y_{1}(k) \\
\vdots \\
y_{n_{p}}(k)
\end{array}\right]}_{y_{i}(k)}=\underbrace{\left[\begin{array}{ccc}
\gamma_{1,1}^{L} & \cdots & \gamma_{1, n_{r}}^{L} \\
\vdots & \ddots & \vdots \\
\gamma_{n_{p}, 1}^{L} & \cdots & \gamma_{n_{p}, n_{r}}^{L}
\end{array}\right]}_{u_{i}^{L}} \\
\underbrace{\left[\begin{array}{c}
u_{1} \\
u_{n_{r}}(k)
\end{array}\right]}_{\Gamma_{i}^{L}(k)}
\end{aligned}
$$

donde $\Gamma_{i}^{L}$ es la matriz de acoplamiento.

Por otro lado, desde el punto de vista de tiempo discreto, el estado del sistema de almacenamiento $x_{s}$ en el tiempo $k+1$ depende del estado del tiempo anterior $k$ y del total de flujo intercambiado $\check{u}_{i, s}^{E}(k)$ durante el periodo $\Delta T$ en el intervalo desde $k$ hasta $k+1$, los valores de flujo de entrada-salida de estado estacionario se describen por la siguiente relación:

$$
\check{u}_{i, s}^{E}(k)=e_{i, s}(k) u_{i, s}^{E}(k)
$$

donde $e_{i, s}(k)$ es la eficiencia de la interfaz $i, s$ de carga y descarga del $H u b$, esta describe el intercambio de energía entre el sistema y el almacenamiento. Este factor depende de la dirección del flujo intercambiado como sigue

$$
e_{i, s}= \begin{cases}e_{i, s}^{+} & \text {si } \quad u_{i, s}^{E} \geq 0 \quad(\text { carga }) \\ e_{i, s}^{-} & \text {otro caso } \quad(\text { descarga })\end{cases}
$$

donde $e_{i, s}^{+}$y $e_{i, s}^{-}$son la eficiencia de carga $\mathrm{y}$ descarga, respectivamente [19]. Asumiendo que $\check{u}$ se mantiene constante durante $\Delta T$, se tiene:

$$
\begin{gathered}
x_{i, s}(k+1)=x_{i, s}(k)+\int_{k}^{k+1} \check{u}_{i, s}^{E}(t) d t \\
=x_{i, s}(k)+\check{u}_{i, s}(k) \Delta T
\end{gathered}
$$

Si se considera que se tienen múltiples sistemas de almacenamiento, entonces se puede representar como:

$$
\begin{aligned}
& \underbrace{\left[\begin{array}{c}
x_{i, 1}(k+1) \\
\vdots \\
x_{i, n_{s}}(k+1)
\end{array}\right]}_{x_{i}(k+1)}=\underbrace{\left[\begin{array}{c}
x_{i, 1}(k) \\
\vdots \\
x_{i, n_{s}}(k)
\end{array}\right]}_{x_{i}(k)} \\
& +\underbrace{\left[\begin{array}{ccc}
e_{i, 1}(k) & & \\
& \ddots & \\
& & e_{i, n_{s}}(k)
\end{array}\right]}_{\Lambda_{i}^{E}(k)} \underbrace{\left[\begin{array}{c}
\check{u}_{i, 1}^{E}(k) \\
\vdots \\
\check{u}_{i, n_{s}}^{E}(k)
\end{array}\right]}_{u_{i}^{E}(k)}
\end{aligned}
$$

Por lo tanto, un $H u b$ puede ser representado de manera condensada por la siguiente expresión:

$$
\begin{aligned}
& x_{i}(k+1)=x_{i}(k)+\Lambda_{i}^{E}(k) u_{i}^{E}(k) \\
& y_{i}(k)=\Gamma_{i}^{L} u_{i}^{L}(k)
\end{aligned}
$$

\section{Modelado del sistema y algoritmo de control}

El modelado de la planta consiste en dos partes: por un lado, de acuerdo a la ecuación (5), la evolución de la variable de estado $S O C_{B A T}$ de la MG se describe por el siguiente modelo lineal:

$$
\begin{aligned}
& S O C_{B A T}(k+1)=S O C_{B A T}(k) \\
& +\frac{\eta_{B A T_{C}} \cdot P_{B A T_{C}}(k) \cdot T_{s}}{C_{B A T}} \\
& +\frac{\eta_{B A T_{D}} \cdot P_{B A T_{D}}(k) \cdot T_{s}}{C_{B A T}}
\end{aligned}
$$

donde $\eta_{B A T_{C}}$ y $\eta_{B A T_{D}}$ son la eficiencia de carga y descarga de las baterías, respectivamente. $T_{s}$ es el tiempo de muestreo, $C_{B A T}$ es la capacidad de la batería y, $P_{B A T_{C}}$ y $P_{B A T_{D}}$ son la potencia de carga y descarga de las baterías en el instante de tiempo $t=k$, respectivamente, y $S O C_{B A T}$ es el estado de carga (State Of Charge) de las baterías.

Por otro lado, se considera una potencia remanente en el instante de tiempo $t=k$ de los subsistemas que integran la MG, ésto es:

$$
P_{r e m}(k)=P_{p v}(k)-C A R G A(k)
$$

donde $C A R G A(k)=P_{C I E}(k)+P_{g h}(k) . \quad P_{p v}$, $P_{C I E}$ y $P_{g h}$ son la energía producida por el sistema $\mathrm{PV}$, la energía consumida por el edificio CIESOL 
y la energía consumida por el invernadero, respectivamente. En la Figura 2 se muestra el diagrama de control.



Figura 2: Micro-red y controlador.

El vector de entrada para la MG se define de acuerdo a:

$$
u=\left[\begin{array}{c}
P_{\text {solar }} \\
P_{R C} \\
P_{R V} \\
P_{B A T_{C}} \\
P_{B A T_{D}}
\end{array}\right]
$$

donde $P_{\text {solar }}$ es la energía solar generada, $P_{R C}$ y $P_{R V}$ son la energía que se compra y se vende a la red eléctrica principal, respectivamente.

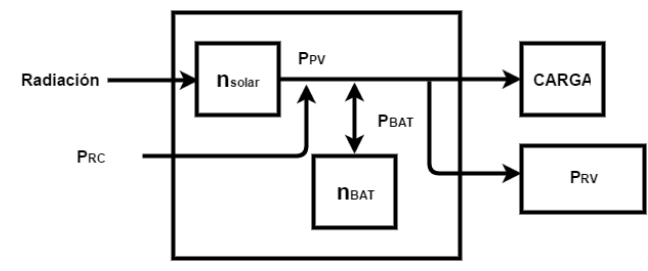

Figura 3: Energy Hub.

Por lo tanto, la MG puede ser modelada como un $H u b$ (ver Figure 3) de manera condensada como sigue:

$$
\begin{aligned}
& x(k+1)=A x(k)+B u(k) \\
& y(k)=D u(k)
\end{aligned}
$$

donde

$$
\begin{aligned}
& A=[1] \\
& B=\left[\begin{array}{lllll}
0 & 0 & 0 & \frac{\eta_{B A T_{C}}}{C_{B A T}} \frac{\eta_{B A T_{D}}}{C_{B A T}}
\end{array}\right] \\
& D=\left[\begin{array}{lllll}
\eta_{\text {solar }} & 1 & -1 & -1 & -1
\end{array}\right]
\end{aligned}
$$

y $\eta_{\text {solar }}$ es la eficiencia de conversión de la energía solar.

El controlador se ha diseñado para lograr la optimización económica de la micro-red y hacer una buena gestión de la utilización de baterías, así también, determinar el momento exacto de la carga. Para el MPC se implementa la siguiente función objetivo:

$$
\begin{aligned}
& J=\sum_{l=0}^{N_{p}-1}\left(\tilde{u}(k+l)^{T} Q_{u} \tilde{u}(k+l)+f^{T} \tilde{u}(k+l)\right) \\
& +\sum_{l=0}^{N_{p}-1}\left(\tilde{x}(k+l)-\tilde{x}_{r e f}(k+l)\right)^{T} Q_{x}(\tilde{x}(k+l) \\
& \left.-\tilde{x}_{r e f}(k+l)\right)
\end{aligned}
$$

sujeto a las siguientes restricciones

$$
\begin{aligned}
\underline{x} & \leq \tilde{x}(k+l+1) \leq \bar{x} \\
\underline{u} & \leq \tilde{u}(k+l+1) \leq \bar{u} \\
\tilde{y}(k+l) & =C A R G A(k) \\
\tilde{u}_{1}(k+l) & =P_{\text {solar }}(k) \\
& =P_{P V \text { disponible }}(k) \\
x(k) & =\check{x}(k)
\end{aligned}
$$

para $l=0, \ldots, N_{p}-1$. Donde $Q_{u}$ y $Q_{x}$ son matrices de peso definidas positivas, $f$ es una función lineal, $N_{p}$ es el horizonte de predicción. En cuanto a la notación " $\tilde{a}$ " sobre las variables, se utiliza para denotar variables sobre el horizonte de predicción, $\underline{a_{i}}$ y $\overline{a_{i}}$ denotan el valor mínimo y máximo permitido y, $\check{a_{i}}$ se refiere a variables en las cuales se supone que sus valores son conocidos, por ejemplo, las condiciones iniciales. Respecto a las restricciones (18) y (19), en éstas se obliga al controlador a suplir la demanda y a utilizar toda la energía generada por el sistema $\mathrm{PV}$, respectivamente.

Para permitir un correcto funcionamiento del sistema respecto a los flujos de energía en las baterías y en el intercambio de energía con la red principal, se han introducido variables binarias como restricciones en la formulación. Estas variables binarias han permitido que en la compra y venta de energía, o en la carga y descarga de las baterías se realice sólo una acción a la vez. La formulación de las variables binarias introducidas se ha realizado de la siguiente manera:

$$
\begin{gathered}
\delta_{R C}(k)= \begin{cases}1, & P_{R C}(k)>0 \\
0, & P_{R C}(k)=0\end{cases} \\
\delta_{R V}(k)= \begin{cases}1, & P_{R V}(k)>0 \\
0, & P_{R V}(k)=0\end{cases} \\
\delta_{R C}(k)+\delta_{R V}(k)=1
\end{gathered}
$$




$$
\begin{gathered}
\delta_{B A T_{C}}(k)= \begin{cases}1, & P_{B A T_{C}}(k)>0 \\
0, & P_{B A T_{C}}(k)=0\end{cases} \\
\delta_{B A T_{D}}(k)= \begin{cases}1, & P_{B A T_{D}}(k)<0 \\
0, & P_{B A T_{D}}(k)=0\end{cases} \\
\delta_{B A T_{C}}(k)+\delta_{B A T_{D}}(k)=1
\end{gathered}
$$

La formulación anterior ha permitido que la formulación del sistema haya sido transformada en una formulación Mixta Lógica Dinámica (MLD), donde se consideran conceptos de calculo proposicional y programación lineal entera como se muestra en [1]. La formulación MLD hace posible la inclusión de variables binarias en un sistema dinámico en tiempo discreto con el fin de describir, en un modelo unificado, la evolución de las señales continuas y la lógica del sistema.

La condición $P_{j}(k)>0$ en las ecuaciones $(22),(21)$ y (24) y la condición $P_{B A T_{D}}<0$ en la ecuación (25) se asocian a las variables binarias $\delta_{j}$ para $j=$ $\left\{R C, R V, B A T_{C}\right\}$ y $\delta_{B A T_{D}}$, respectivamente, esto es

$$
\begin{gathered}
{\left[P_{j}(k)>0\right] \rightarrow\left[\delta_{j}(k)=1\right]} \\
{\left[P_{B A T_{D}}<0\right] \rightarrow\left[\delta_{B A T_{D}}(k)=1\right]}
\end{gathered}
$$

La formulación (27) se expresa por las siguientes desigualdades

$$
\begin{gathered}
(M+\epsilon) \delta_{j}(k)-P_{j} \delta_{j}(k)>0 \\
(m+\epsilon) \delta_{B A T_{D}}(k)-P_{B A T_{D}} \delta_{B A T_{D}}(k)<0
\end{gathered}
$$

donde

$$
\begin{aligned}
& m \quad \min P_{B A T_{D}}(k) \\
& M \quad \max P_{j}(k)
\end{aligned}
$$

y $\epsilon$ es una pequeña tolerancia (típicamente la precisión del computador).

En la ecuación $(21)$, cuando $P_{R C}(k)>0$ se tiene que $\delta_{R C}(k)=1$, esto significa que la energía es comprada a la red y por lo tanto el peso de compra es usado, en (22), cuando $P_{R V}(k)>0$ se tiene que $\delta_{R V}(k)=1$, esto significa que la energía es vendida a la red y por lo tanto el peso de venta es usado. De manera análoga se realiza el análisis para las ecuaciones (24) y (25). Además, las ecuaciones (23) y (26) se asocian a la suma exclusiva (or exclusivo) de la manera siguiente

$$
\begin{gathered}
{\left[P_{R C}(k) \oplus P_{R V}(k)\right]} \\
{\left[P_{B A T_{C}}(k) \oplus P_{B A T_{D}}(k)\right]}
\end{gathered}
$$

ésto significa que para la compra y venta de energía con la red, y para la carga y descarga de energía de las baterías, solamente una acción puede ser realizada a la vez, compra o venta de energía y carga o descarga de las baterías.

\section{Resultados}

Los resultados que se muestran a continuación han sido obtenidos a partir de datos reales recolectados durante los días 17 y 19 (uno soleado y uno con nubes) de marzo de 2015. Se ha considerado el consumo eléctrico de solamente un laboratorio del edificio CIESOL. En las Figuras 4 y 5 se muestran la producción y consumos de los sistemas CIESOL, invernadero y PV para los días 17 y 19 de marzo de 2015.

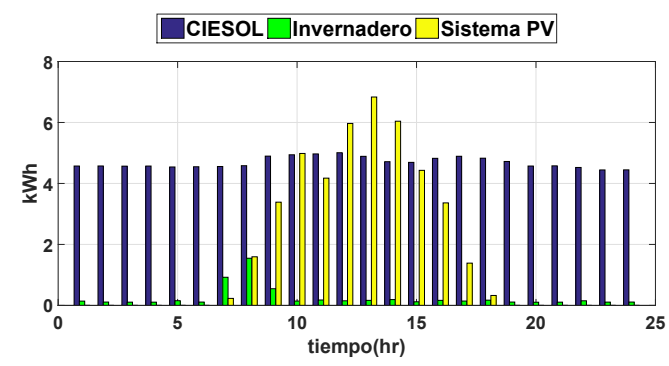

Figura 4: Producción del sistema PV y consumos de los sistemas CIESOL e invernadero durante el día 17 de marzo de 2015.

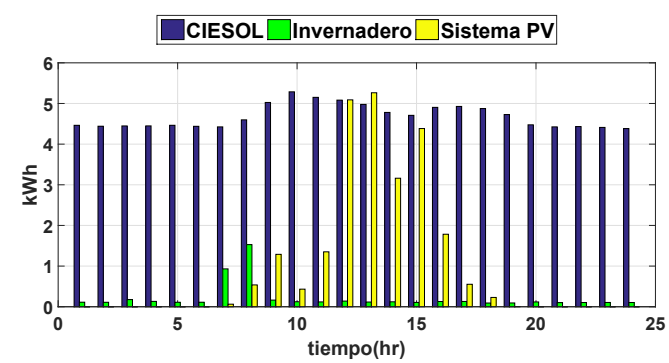

Figura 5: Producción del sistema PV y consumos de los sistemas CIESOL e invernadero durante el día 19 de marzo de 2015.

Durante el día 17 de marzo de 2015 el edificio CIESOL ha consumido un total de $112.45 \mathrm{kWh}$, el invernadero ha consumido $5.87 \mathrm{kWh}$, esto es, se he tenido una demanda total de $118.32 \mathrm{kWh}$, y el sistema PV ha producido $42.72 k W h$. Por otro lado, durante el día 19 de marzo de 2015, el edificio CIESOL y el invernadero han consumido $111.29 k W h$ y $5.07 k W h$, respectivamente, esto es, se ha tenido una demanda de $117.36 \mathrm{kWh}$, el sistema PV ha producido $24.14 k W h$. La energía producida por el sistema PV no ha sido sufiente para suplir la demanda de cada día, por lo tanto, 
se ha tenido que comprar a la red principal un total de $75.61 k W h$ y $93.23 k W h$ para los días 17 y 19 de marzo, respectivamente.

Con la finalidad de verificar el comportamiento y los beneficios del controlador que se ha propuesto, se ha realizado una simulación para los días 17 y 19 de marzo de 2015. El primer término de la función objetivo (ecuación (15)) se ha utilizado para la gestión de las fuentes renovables y la compra/venta de energía de la red. La matriz de peso $Q_{u}$ y la función $f$ han sido sintonizadas de acuerdo al coste de cada fuente de energía. En el segundo término, $Q_{x}$ ha sido sintonizada para mantener un nivel de carga al rededor de 40\%, así también, se ha permitido desviaciones al rededor de $40 \%$ para cuando se necesite almacenar más energía o utilizar la energía almacenada. Con la finalidad de que se haga un mayor uso de las fuentes de energía renovables y que las baterías tengan un menor desgaste, se ha asignado a la matriz $Q_{x}$ un valor menor al que se ha asignado a la matriz $Q_{u}$.

El controlador que se propone ha sido aplicado a la micro-red. Todas las cantidades se expresan en $k W h$. El principal objetivo del controlador es maximizar el uso de energías renovables y optimizar la compra y venta de energía con la red principal. Con la finalidad de comparar costos, se ha considerado un día soleado y un día con nubes, ambos con una demanda de energía similar. Se ha considerado el mismo precio de compra y venta de energía para ambos días (ver Figura 12). El controlador ha sido implementado en MATLAB [18] y se ha hecho uso del toolbox YALMIP [17] y el solver CPLEX [13]. En las Figuras 6 - 8 y 9 - 11 se muestran los resultados de las simulaciones para un día soleado y un día con nubes, respectivamente. En las Figuras 6 y 9 se muestran la cantidad de energía solar disponible, así como la cantidad de energía utilizada para cargar y descargar la batería, además, se muestra el flujo de energía entre la micro-red y la red principal. El estado de carga de las baterías para el día soleado y día con nubes se muestra en las Figuras 7 y 10, respectivamente. En las Figuras 8 y 11 se puede observar que el controlador proporciona a la micro-red la energía total demandada, se puede observar que la demanda de energía de ambos días son similares.

Por otro lado, se puede observar en la Figura 6 que durante el periodo desde las 9 horas hasta las 15 horas, en la micro-red sólo se ha usado la energía proporcionada por el sistema PV y las baterías, por lo tanto, no se ha permitido la compra de energía a la red principal. Además, se puede observar que en el periodo desde las 11 horas hasta las 14 horas se ha permitido vender energía a la

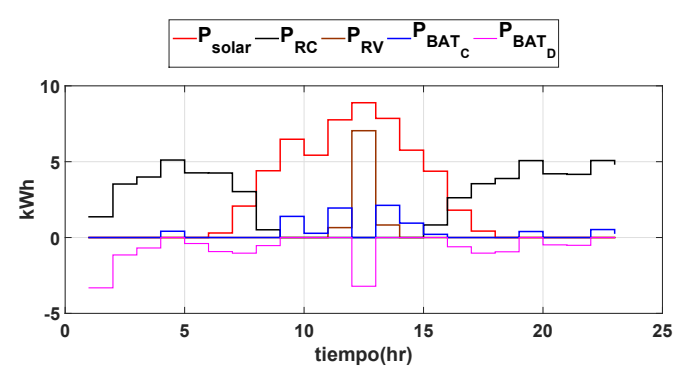

Figura 6: Resultados del controlador propuesto (día soleado).

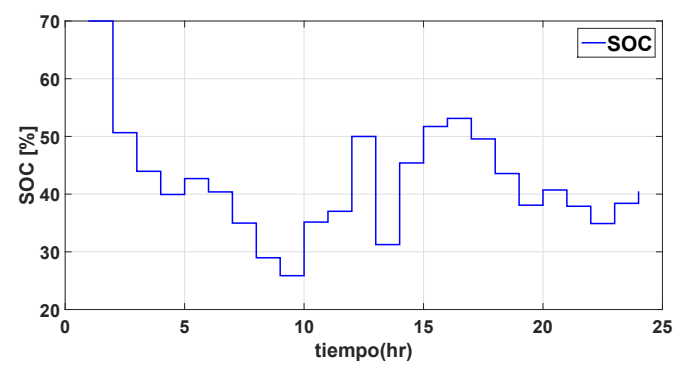

Figura 7: Estado de carga de las baterías (día soleado).

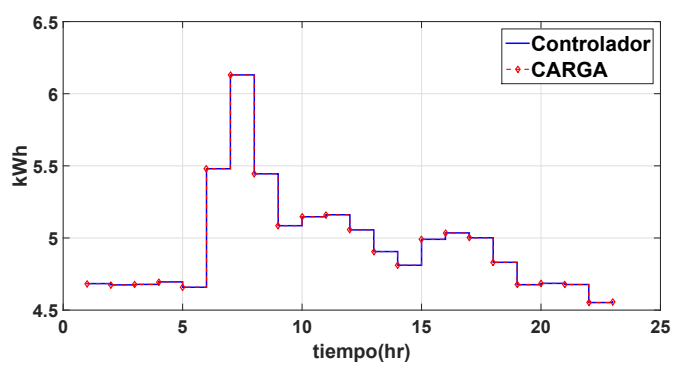

Figura 8: Potencia demandada por la carga y suplida por el controlador (día soleado).

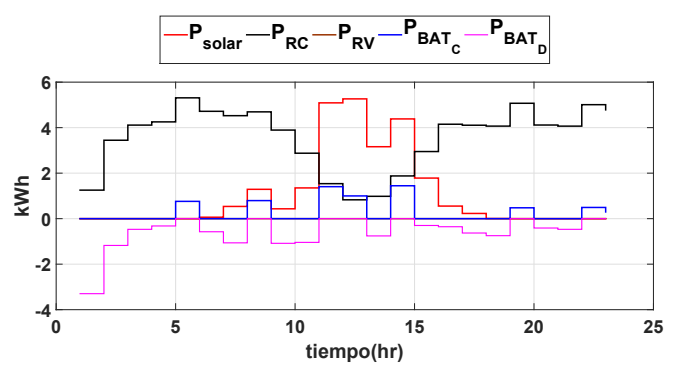

Figura 9: Resultados del controlador propuesto (día con nubes).

micro-red. Aunque el controlador ha intentado mantener el estado de carga de las baterías alrededor del $40 \%$ (ver Figuras 7 y 10), se ha permitido fluctuaciones en los momentos donde las energías renovables no han sido capaces de suplir la energía demandada. En el caso del día con nubes, se 


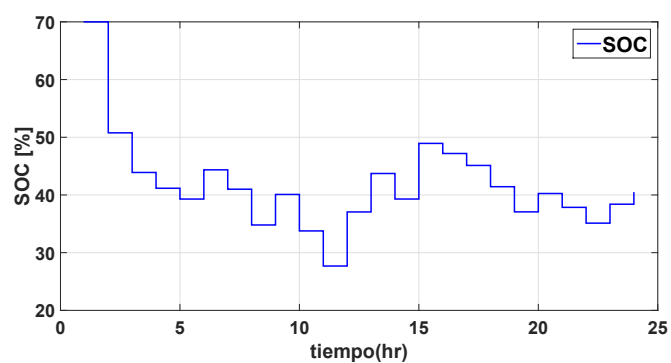

Figura 10: Estado de carga de las baterías (día con nubes).

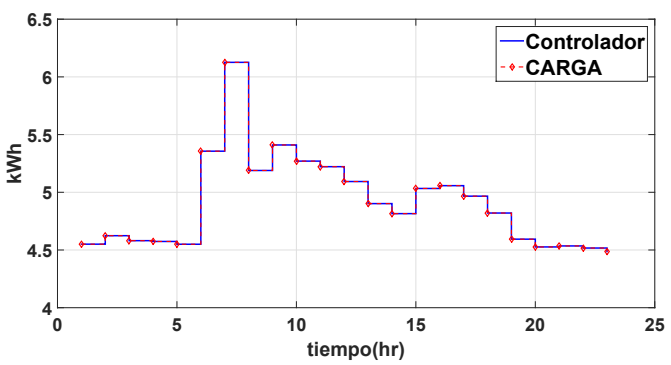

Figura 11: Potencia demandada por la carga y suplida por el controlador (día con nubes).

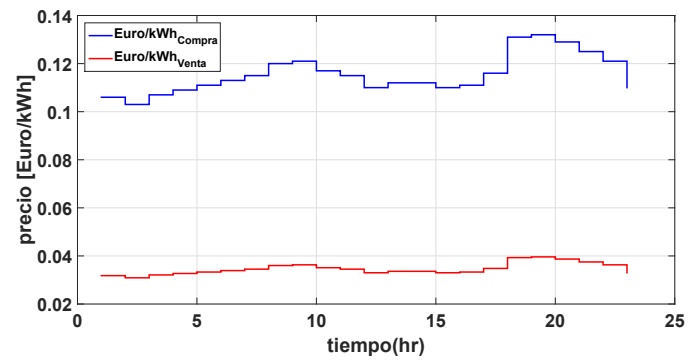

Figura 12: Precios de compra y venta de energía.

Tabla 1: Energía intercambiada con la red principal usando el controlador.

\begin{tabular}{lcc}
\hline \multicolumn{3}{c}{ Día soleado } \\
\hline Energía $(k W h)$ & Euros $(€)$ \\
\hline Vendida & 8.521 & 0.942 \\
Comprada & 60.309 & 2.117 \\
\hline \multicolumn{3}{c}{ Día con nubes } \\
\hline Energía $(k W h)$ & Euros $(€)$ \\
\hline Vendida & 0 & 0 \\
Comprada & 82.624 & 2.889 \\
\hline
\end{tabular}

puede observar que el controlador no ha permitido la venta de energía (ver Figura 9).

En la Tabla 1 se muestran los resultados de la energía intercambiada con la red principal y el costo de este intercambio, se puede observar que para el día soleado ha sido comprado un $79.76 \%$ de la energía total demandada, esto es, se ha po- dido conseguir un ahorro de $20.24 \%$, además de este ahorro, el controlador ha permitido vender energía a la red. Para el día con nubes se ha podido conseguir un ahorro de $11.38 \%$. El coste de degradación de las baterías y el coste de mantenimiento del sistema PV no han sido considerados.

\section{Conclusiones y trabajo futuro}

En este artículo se ha propuesto un controlador que optimice el uso de energías renovables en una micro-red. Se ha considerado las baterías del vehículo eléctrico como un sistema de almacenamiento. Por una parte, el controlador que ha sido propuesto ha permitido fluctuaciones de carga y descarga en las baterías al rededor de un valor deseado con la finalizar de optimizar el uso de energías renovables. Por otro lado, el precio de compra y venta de energía ha sido considerado variable a lo largo del tiempo. El esquema de control que se ha propuesto ha presentado resultados favorables. Los resultados han sido derivados de simulaciones realizadas durante dos días (un día soleado y un día con nubes). Para futuros trabajos se introducirán modelos de predicción basados en series temporales y redes neuronales para la demanda de energía de los sistemas de carga, producción de energía del sistema PV y en la predicción de los precios de la energía. Además, se realizará una comparación económica con la finalidad de verificar los beneficios que puedan otorgar los modelos de predicción al controlador que ha sido propuesto en este trabajo.

\section{Agradecimientos}

Este trabajo ha sido financiado con el Proyecto $\mathrm{R}+\mathrm{D}+\mathrm{i}$ del Plan Nacional DPI2014-56364-C2-1$\mathrm{R}$ del Ministerio de Economía y Competitividad y Fondos FEDER.

\section{Referencias}

[1] Bemporad, A., And Morari, M. Control of systems integrating logic, dynamics, and constraints. Automatica 35, 3 (1999), 407427.

[2] Camacho, E. F., And Alba, C. B. Model predictive control. Springer Science \& Business Media, 2013.

[3] Carmeli, M. S., Castelli-Dezza, F., Mauri, M., Marchegiani, G., AND Rosati, D. Control strategies and configurations of hybrid distributed generation systems. Renewable Energy 41 (2012), 294-305.

[4] Chitsaz, H., Shaker, H., Zareipour, H., WoOd, D., AND AMJAdY, N. Short-term 
Electricity Load Forecasting of Buildings in Microgrids. Energy and Buildings 99 (apr 2015), 50-60.

[5] Dursun, E., And Kilic, O. Comparative evaluation of different power management strategies of a stand-alone pv/wind/pemfc hybrid power system. International Journal of Electrical Power \& Energy Systems 34, 1 (2012), 81-89.

[6] Eghtedarpour, N., And Farjah, E. Control strategy for distributed integration of photovoltaic and energy storage systems in dc micro-grids. Renewable Energy 45 (2012), 96-110.

[7] Etxeberria, A., Vechiu, I., Camblong, H., AND Vinassa, J.-M. Hybrid energy storage systems for renewable energy sources integration in microgrids: A review. In $I P E C$, 2010 Conference Proceedings (2010), IEEE, pp. $532-537$.

[8] Ferrari-Trechte, G., Gallestey, E., Letizia, P., Spedicato, M., Morari, M., And Antonine, M. Modeling and control of co-generation power plants: a hybrid system approach. IEEE Transactions on Control Systems Technology 12, 5 (2004), 694-705.

[9] Geidl, M., And Andersson, G. A modeling and optimization approach for multiple energy carrier power flow. In Power Tech, 2005 IEEE Russia (2005), IEEE, pp. 1-7.

[10] Geidl, M., Koeppel, G., Favre-Perrod, P., Klockl, B., Andersson, G., And Frohlich, K. Energy hubs for the future. IEEE Power and Energy Magazine 5, 1 (2007), 24.

[11] Gu, W., Wu, Z., And Yuan, X. Microgrid economic optimal operation of the combined heat and power system with renewable energy. IEEE Power and Energy Society General Meeting (2010), 1-6.

[12] Huang, W., Lu, M., And Zhang, L. Survey on microgrid control strategies. Energy Procedia 12 (2011), 206-212.

[13] ILOG. Cplex.

[14] Indu Rani, B., Saravana Ilango, G., And Nagamani, C. Power flow management algorithm for photovoltaic systems feeding dc/ac loads. Renewable Energy 43 (2012), $267-275$.

[15] Karabiber, A., Keles, C., Kaygusuz, A., AND Alagoz, B. B. An approach for the integration of renewable distributed generation in hybrid dc/ac microgrids. Renewable Energy 52 (2013), 251-259.

[16] Kurohane, K., Uehara, A., Senjyu, T., Yona, A., Urasaki, N., Funabashi, T., AND KIM, C.-H. Control strategy for a distributed dc power system with renewable energy. Renewable Energy 36, 1 (2011), 42-49.

[17] LofberG, J. Yalmip : A toolbox for modeling and optimization in matlab. In $C A C S D$ Conference, Taipei, Taiwan. (2004).

[18] MATLAB. version 8.4.0 (R2014b). The MathWorks Inc., 2014.

[19] Mendes, P. R. D. C., Normey-Rico, J. E., AND Alba, C. B. Economic energy management of a microgrid including electric vehicles. In Innovative Smart Grid Technologies Latin America (ISGT LATAM), 2015 IEEE PES (2015), IEEE, pp. 869-874.

[20] Morais, H., Kádár, P., Faria, P., Vale, Z. A., AND KHODR, H. Optimal scheduling of a renewable micro-grid in an isolated load area using mixed-integer linear programming. Renewable Energy 35, 1 (2010), 151-156.

[21] Nehrir, M., Wang, C., Strunz, K., Aki, H., Ramakumar, R., Bing, J., Miao, Z., AND SAlameH, Z. A review of hybrid renewable/alternative energy systems for electric power generation: Configurations, control, and applications. Sustainable Energy, IEEE Transactions on 2, 4 (2011), 392-403.

[22] Rodríguez, F., Berenguel, M., Guzmán, J. L., AND RAmíREz-Arias, A. Modeling and Control of Greenhouse Crop Growth. Advances in Industrial Control. Springer International Publishing, Cham, 2015.

[23] Sánchez-Molina, J. A., Reinoso, J. V., Acién, F. G., Rodríguez, F., And López, J. C. Development of a biomass-based system for nocturnal temperature and diurnal CO2 concentration control in greenhouses. Biomass and Bioenergy 67 (2014), 60-71.

[24] Valverde, L., Bordons, C., And Rosa, F. Power management using model predictive control in a hydrogen-based microgrid. Annual Conference on IEEE Industrial Electronics Society (2012), 5669-5676. 